\title{
Buckling of a spherical shell under external pressure and inward concentrated load: asymptotic solution
}

\author{
A. Evkin \\ Software for Structures, Toronto, L4J 8E6, Canada
}

\section{Kolesnikov}

Pridneprovska State Academy of Civil Engineering and Architecture, Dnipropetrovsk, 49600, Ukraine

\section{A. Prikazchikov ${ }^{1}$}

School of Computing and Mathematics, Keele University, Staffordshire, ST5 5BG, UK

\begin{abstract}
An asymptotic solution is suggested for a thin isotropic spherical shell subject to uniform external pressure and concentrated load. The pressure is the main load and a concentrated lateral load is considered as a perturbation that decreases buckling pressure. First, the post-buckling solution of the shell under uniform pressure is constructed. A known asymptotic result for large deflections is used for this purpose. In addition, an asymptotic approximation for small post-buckling deflections is obtained and matched with the solution for large deflections. The proposed solution is in good agreement with numerical results. An asymptotic formula is then derived, with the load-deflection diagrams analyzed for the case of combined load. Buckling load combinations are calculated as limiting points in the load-deflection diagrams. The sensitivity of the spherical shell under external pressure to local perturbations is analyzed. The suggested asymptotic result is validated by finite element method using ANSYS simulation software package.
\end{abstract}

\section{Keywords}

Spherical shell, post-buckling behaviour, uniform asymptotic, load combination, perturbation analysis.

\section{Introduction}

Thin shell structures find wide applications in many branches of engineering. Examples include aircrafts, spacecrafts, nuclear reactors, tanks for liquid and gas storage, and pressure vessels. Engineers have to reduce the weight and cost of the structures, reducing their thickness and applying advanced materials and technologies. This is especially important for aircrafts and launch vehicles. Cylindrical and spherical shells are often used as elements in such structures.

Because of the thinness of the structures, buckling is the most common failure mode. The classical buckling pressure of a complete elastic thin spherical shell was obtained by R. Zoelly [1], for more details see also [2]

$$
q_{c}=\frac{2 E}{\beta} \frac{h^{2}}{R^{2}} .
$$

Here $E$ is the Young modulus, $h$ and $R$ are the thickness and radius of the shell's mid-surface, respectively, and

$$
\beta=\sqrt{3\left(1-\nu^{2}\right)}
$$

\footnotetext{
${ }^{1}$ Corresponding author: School of Computing and Mathematics, Keele University, Staffordshire, ST5 5BG, UK. Email: d.prikazchikov@keele.ac.uk
} 
with $\nu$ denoting the Poisson's ratio.

It should be noted that the classical result (1.1) is obtained in assumption that the shell is geometrically perfect.

However, in reality presence of various external and internal perturbations including geometrical imperfections can reduce the buckling load to a small fraction of the classical value. This is illustrated by Fig. 1, where the ratio of experimental critical load values $q_{\exp }$ to the classical $q_{c}$ are plotted versus $R / h$, see [3]. As described in the above-cited article, the limiting curve presented in [4], corresponds to the lower bound for most of the known experimental results. It may be observed from the graph that as the shell becomes thinner, the ratio $q_{e x p} / q_{c}$ decays so the classical results need refinement. As established by Koiter [5], extreme sensitivity of the structure occurs due to its non-linear post-buckling behavior.

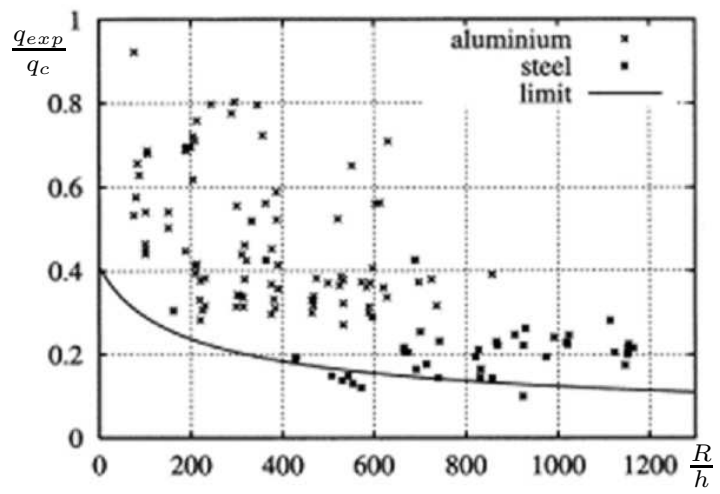

Figure 1: Experimental results for spherical shells under external pressure, published in [3].

Perturbations reducing the critical buckling load, can be classified by their nature, including initial deviation from perfect spherical shape of the shell (geometrical imperfections), additional load or deflections caused by the load, and perturbations characterized by energy which can lead to shell buckling. Lateral inward-directed point load is the most common factor among external perturbations decreasing buckling value of the main load. In the present research we study the effect of additional concentrated load and deflections considering the shell of perfect spherical shape.

A simple solution for combination of external pressure and concentrated load has been obtained by Sabir [6]. The author considered two regions: the dimple, or inner region, and the rest of the shell, or outer region. It is assumed that each region suffers small displacements from a spherical surface, namely the unstrained surface for the outer region, and a surface of equal but reversed curvature for the inner region. However, the obtained result is not accurate enough because of the assumption that the equilibrium state can be described by linear equations. The nonlinear behavior of a shell under concentrated load has been investigated, for example, in $[7,8]$. As shown in later works, the buckling loads calculated in [6] exceed significantly the results obtained in experiments [9] and in the numerical analysis presented below in the paper. In this paper we will employ the asymptotic methodology described in [10].

The aim of this paper is to obtain a simple but accurate enough approximate solution for further analysis of the effect of perturbations on the buckling pressure. The obtained formulae may also be used for estimation of buckling loads at the design state of a real structure under considered load combination.

The paper is organized as follows. The post-buckling solution of the shell under uniform external pressure is obtained in Section 2, starting from the nonlinear shallow-shell equations describing rotationally symmetric deformations. An asymptotic solution obtained in [11] for large deflections is described. In addition, an approximate formula for small post-buckling deflections is obtained and matched with the solution for large deflections. In Section 3 this approach is generalized to the case of a combined load. The obtained analytical results are validated numerically. Section 4 contains description of the ANSYS shell model, main results of the calculations, and their discussion. 


\section{Post-buckling behavior of perfect shell under external pres- sure}

\subsection{Shallow shell equations}

The nonlinear shallow-shell equations will be employed below, relying on the assumption that the size of buckling and post-buckling section of the shell surface is small compared to its radius. We consider rotationally symmetric deformations assuming that boundary conditions will not affect the result of the analysis. From the previous studies [10] it is known that the assumption is justified provided that the concentrated load is applied at the distance of about $2 \sqrt{R h}$ from the shell boundary. The governing system of equations including one equilibrium equation and one compatibility equation can be written in terms of the normal displacement $W$ and the stress function $F$, namely

$$
\begin{aligned}
& \frac{D}{h} \nabla^{4} W=\frac{1}{r} \frac{d}{d r}\left(\frac{d W}{d r} \frac{d F}{d r}\right)+\frac{1}{r R} \frac{d}{d r}\left(r \frac{d F}{d r}\right)+\frac{q}{h} \\
& \frac{1}{E} \nabla^{4} F+\frac{1}{r} \frac{d^{2} W}{d r^{2}} \frac{d W}{d r}+\frac{1}{r R} \frac{d}{d r}\left(r \frac{d W}{d r}\right)=0
\end{aligned}
$$

where $D=E h^{3} / 12\left(1-\nu^{2}\right)$ is the bending stiffness, $\nabla^{4}$ is the biharmonic operator in the polar coordinate $r$ (see Fig. 2), and $q$ is external pressure, see [2].

\section{$2.2 \quad$ Large deflections}

In order to introduce the methodology in this subsection we describe the idea and present the main results of an asymptotic approach suggested in [11] for thin isotropic spherical shell experiencing large deflections compared to shell thickness. The complete asymptotic analysis for large deflections of a shell of revolution has been presented in $[12,13]$.

The consideration is based on the parameter

$$
\varepsilon^{2}=\frac{2 h}{w_{0} \beta}
$$

which has been introduced in [11], being proportional to the ratio of shell thickness $h$ to the deflection amplitude $w_{0}$. It is small for deflections which are large compared to shell thickness.

On introducing the scaling

$$
z=\frac{r^{2}}{R w_{0}}, \quad w=\frac{W}{w_{0}}, \quad \phi=\frac{2 F \beta}{E h w_{0}}, \quad \bar{q}=\frac{q}{q_{c}},
$$

the parameter $\varepsilon^{2}$ appears at highest order derivatives in the equations

$$
\begin{aligned}
& \varepsilon^{2} \frac{d^{2}}{d z^{2}}\left(z \frac{d w}{d z}\right)=\frac{d \phi}{d z}\left(1+2 \frac{d w}{d z}\right)+\bar{q}, \\
& \varepsilon^{2} \frac{d^{2}}{d z^{2}}\left(z \frac{d \phi}{d z}\right)=-\frac{d w}{d z}\left(1+\frac{d w}{d z}\right) .
\end{aligned}
$$

At leading order $(\varepsilon=0)$ we have two solutions

1) $\frac{d w}{d z}=0 \quad$ or $\quad \frac{d w}{d r}=0$;

2) $1+\frac{d w}{d z}=0 \quad$ or $\quad \frac{d^{2} w}{d r^{2}}=-\frac{2}{R}$.

The first one corresponds to the initial trivial state of the shell (region $F_{1}$ in Fig. 2). The second one (region $F_{2}$ ) is a reflection of a part of initial mid-surface of the shell with respect to plane A, which is normal to the rotation axis. This is pure bending of the shell in absence of membrane stresses (midsurface isometric transformation). The main deformation energy of the shell with large deflections is 
concentrated in a narrow zone (inner boundary layer) smoothly connecting the regions $F_{1}$ and $F_{2}$, which can be calculated separately. In contrast, the work of external pressure and concentrated load applied at the shell pole does not depend on the boundary layer deformation in terms of the first order asymptotic analysis. It is defined by inverted part $F_{2}$.

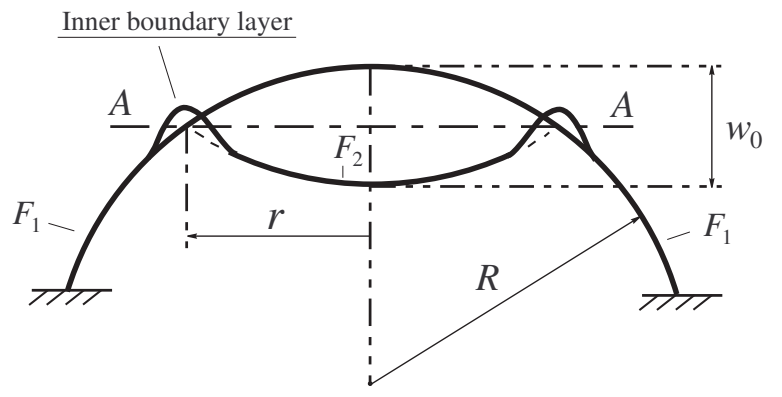

Figure 2: Spherical shells with large deflections.

The solution may be presented as follows

$$
w=w^{0}(z)+\varepsilon w_{1}\left(\frac{1-z}{\varepsilon}\right), \quad \phi=\phi^{0}(z)+\varepsilon \phi_{1}\left(\frac{1-z}{\varepsilon}\right),
$$

where the first terms correspond to isometric transformation of the shell mid-surface, whereas the rapidly changing boundary layer functions $w_{1}$ and $\phi_{1}$ are smoothly connecting $F_{1}$ and $F_{2}$. The nonlinear coupled equations for the boundary layer functions are given by

$$
w_{1}^{\prime \prime \prime}-\phi_{1}^{\prime}\left(1-2 w_{1}^{\prime}\right)=0, \quad \phi_{1}^{\prime \prime \prime}+w_{1}^{\prime}\left(1-w_{1}^{\prime}\right)=0,
$$

subject to the following boundary conditions

$$
\begin{aligned}
& w_{1}^{\prime}=0, \quad \phi_{1}^{\prime}=0, \quad \text { as } \quad t \rightarrow-\infty, \\
& w_{1}^{\prime}=1, \quad \phi_{1}^{\prime}=0, \quad \text { as } \quad t \rightarrow+\infty .
\end{aligned}
$$

Here the derivatives are taken with respect to the "fast" variable $t=(1-z) / \varepsilon$.

It is remarkable that the boundary value problem $(2.6),(2.7)$ does not depend on shell parameters, load level and deflection amplitude. This is due to the new variable $z$ introduced in (2.3). It should be emphasized that the inner boundary layer is always located in the vicinity of $z=1$ independently of load and deflection amplitude. Therefore the boundary layer problem should be solved once, and the result will be valid for different values of load, deflection amplitude and shell parameters. Now it can be solved numerically.

The relationship between the load and the deflection amplitude is established by variation of total potential energy with respect to parameter $\varepsilon$ (or deflection amplitude). The boundary layer deformation energy is defined as

$$
U=D_{1} J_{0} \varepsilon
$$

where

$$
D_{1}=\frac{4 \pi D}{R \beta \varepsilon^{4}},
$$

and

$$
J_{0}=4 \int_{-\infty}^{\infty}\left[\left(w_{1}^{\prime \prime}\right)^{2}+\left(\phi_{1}^{\prime \prime}\right)^{2}\right] d t \approx 2.23
$$


Finally, the following asymptotic approximation for $\bar{q}$ has been derived in [11] for the shell under uniform external pressure

$$
\bar{q}=\frac{3}{16} \varepsilon J_{0}+O\left(\varepsilon^{3}\right) .
$$

A similar result with an additional multiplier of $\left(1-\nu^{2}\right)^{1 / 4}$ has been obtained in [14], however involving initial geometrical assumptions (isometric transformation) of the shell's mid-surface for large deflections.

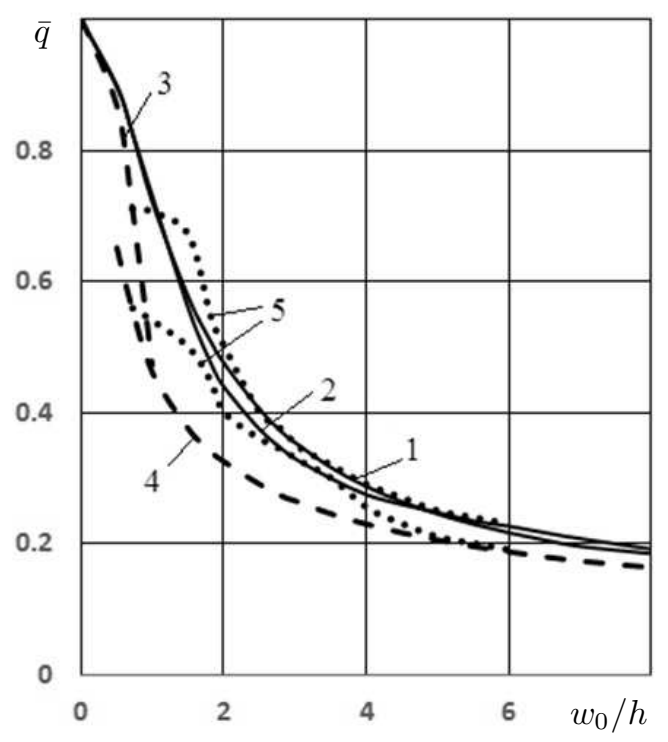

Figure 3: Post-buckling behavior of spherical shell (asymptotic, numerical and experimental results).

In Fig. 3 the precise experimental results [15] are represented by dotted lines 5 . They yield upper and lower bounds of obtained data. The shell specimens were manufactured from melded copper which evaporated in vacuum and condensed on the ceramic substrate of the spherical form. This methodology allowed to minimize influence of the imperfections, which was quite moderate according to Fig. 3 for $\bar{q} \leq$ 0.5 , increasing only at external pressure parameter of about 0.6.

The dashed curve 4 corresponding to the asymptotic formula (2.11) is compared with experimental results (curves 5), and numerical solutions $[16,17]$ depicted by solid line 2 , calculated for the complete spherical shell with the ratio $R / h=100$. A good agreement between experimental and numerical results is observed, however asymptotic formula (2.11) yields good approximation only for large deflection amplitude. The curves 1 and 3 will be described later in this section and correspond to the proposed approximations.

\subsection{Initial post-buckling analysis}

In the previous section 2.2 we described the solution obtained in [11] which is valid for large deflections. Since our goal in the present paper is an asymptotic formula for the entire range of deflection amplitude, in this section we derive the solution for small deflections. Below we implement the asymptotic form of solution suggested by Koiter [5]

$$
\bar{q}=1+a\left(\frac{w_{0}}{h}\right)+b\left(\frac{w_{0}}{h}\right)^{2},
$$

where $a$ and $b$ are the coefficients defining post-buckling curve in the vicinity of classical critical load. If they are negative, the curve is decreasing and the structure is sensitive to imperfections. Using the assumption that the deformations are rotationally symmetric, Thompson [18] has shown that the slope of the pressure-deflection curve is negative

$$
a=-\frac{3 \sqrt{6 \beta}}{4 \pi}\left(\frac{h}{R}\right)^{1 / 2} .
$$


However the coefficient $a$ is small for thin shells as $h / R \ll 1$, and can be neglected in our asymptotic analysis. Usually it is relatively easy to calculate the coefficient $a$ because it is defined by classical buckling mode of the structure. Evaluation of the coefficient $b$ is a much more complicated problem, especially for thin shells having numbers of buckling modes corresponding to a set of very close eigenvalues (the buckling loads). The spherical shell under external pressure is the case, and the coefficient $b$ has not been calculated yet for this structure due to the complexity of the problem.

In order to define the initial post-buckling behaviour of the shell we suggest considering a special intermediate equilibrium state, when the curvature of the shell surface at the pole of the sphere is equal to 0 . This state can be defined as

$$
\frac{d^{2} W}{d r^{2}}+\frac{1}{R}=0 \quad \text { at } \quad r=0
$$

In addition we have the symmetry condition $\frac{d W}{d r}=0$ at the pole of the sphere, and therefore the equations (2.1) decouple. The first equation may be simplified to the form of the plate bending equation

$$
D \nabla^{4} W=q
$$

It can be considered as a limiting form of the original equations (2.1) as $r \rightarrow 0$ for the specific equilibrium state, which is really intermediate in terms of curvature change, because for pre-buckling state we have $\frac{d^{2} W}{d r^{2}}=0$, for the shell with large deflections $\frac{d^{2} W}{d r^{2}}=-\frac{2}{R}$ (inverted sphere) and from (2.14) we have the intermediate value $\frac{d^{2} W}{d r^{2}}=-\frac{1}{R}$. Introducing the scaling

$$
x=\frac{r}{\sqrt{R h}}, \quad \text { and } \quad \bar{w}=\frac{W}{h},
$$

we obtain from (2.15) the following expansion as $x \rightarrow 0$

$$
\bar{w}(x)=\frac{w_{0}}{h}-\frac{x^{2}}{2}+\frac{\bar{q} \beta x^{4}}{8}+O\left(x^{6}\right) .
$$

On the other hand, deformations of the shell are diminishing as $x \rightarrow \infty$, therefore we can linearize equations (2.1) for large $x$, with the approximate solution given by

$$
\bar{w}(x)=e^{-x \sqrt{\beta(1-\bar{q})}} \frac{A \cos (x \sqrt{\beta(1+\bar{q})})+B \sin (x \sqrt{\beta(1+\bar{q})})}{\sqrt{x}}
$$

If $\bar{q}=1$, equation (2.18) is an asymptotic representation of Bessel function $(x \rightarrow \infty)$, which is a buckling mode of a perfect spherical shell, see e.g. [2].

An approximate solution suitable for entire range of variable $x$ may be found in the form

$$
\bar{w}(x)=\frac{w_{0}}{h} \frac{\sin (x \sqrt{\beta(1+\bar{q})})\left(1+x^{6}\right)^{1 / 12}}{x \sqrt{\beta(1+\bar{q})} \cosh (x \sqrt{\beta(1-\bar{q})})} .
$$

Indeed, in view of the symmetry, this function is even and it has the same representation as (2.18) when $x \rightarrow \infty$. At the same time its expansion as $x \rightarrow 0$ should coincide with (2.17). The power 6 in the the term $\left(1+x^{6}\right)^{1 / 12}$ is taken for the sake of simplicity, because it can be omitted while deriving the expansion of (2.19) when $x \rightarrow 0$. On the other hand, the power $1 / 12$ is chosen in order to provide $\left(1+x^{6}\right)^{1 / 12} / x \sim x^{-1 / 2}$ as $x \rightarrow \infty$. We also note the approximation for the intermediate point

$$
\bar{q}=0.732, \quad \frac{w_{0}}{h}=\frac{1.18}{\beta}
$$

of the post-buckling load-deflection curve, following from (2.17). According to (2.20), $w_{0} \approx 0.71 \mathrm{~h}$ for $\nu=0.3$, therefore the obtained solution $(2.17)$ is an intermediate nonlinear one. For small deflections the 
solution for the spherical shell under external pressure is given by the classical buckling mode (expressed through a Bessel function). For large deflections the solution is close to isometric transformation of the middle shell surface with an inner boundary layer (Fig. 2).

The corresponding deflection function (2.19) is shown in Fig. 4 by solid line. Numerical solution [19] is represented here by dashed line for comparison $(R / h=100)$.

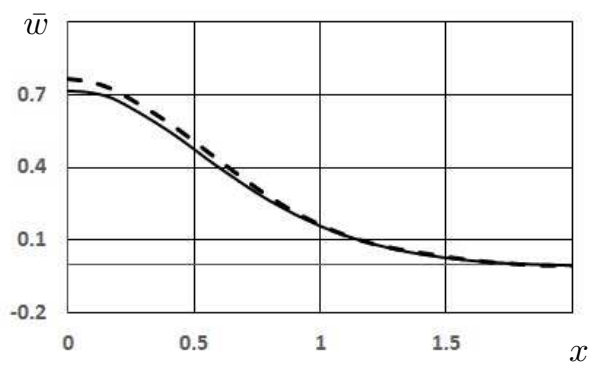

Figure 4: Deflection diagram at intermediate post-buckling shell stage.

The function (2.12) is passing through the obtained intermediate point if $b=-0.58\left(1-\nu^{2}\right)$, yielding the explicit formula

$$
\bar{q}=1-0.58\left(1-\nu^{2}\right) \frac{w_{0}^{2}}{h^{2}}
$$

or

$$
\bar{q}=1-0.77 \varepsilon^{-4},
$$

with corresponding curve 3 in Fig. 3. Formula (2.22) showing a good approximation of the post-buckling shell behavior for small deflections $(\bar{q} \geq 0.8)$.

We would like to emphasize that we used the Koiter's form of the post-buckling solution representation but not the post-buckling theory itself. However, obtained approximation (2.22) for small deflections is good enough for our purposes.

\subsection{Padé approximation}

It is remarkable that both solutions (2.11) and (2.22) (curves 4 and 3 in Fig. 3, respectively) do not depend on shell parameters therefore we can join them to obtain an approximate solution for the entire range of defection amplitude. For techniques of matching different asymptotic solutions the reader is referred to $[20,21]$. Below we employ the two-point Padé functions [22] for this purpose. They are rational functions

$$
\bar{q}(\varepsilon)=\frac{\alpha_{0}+\sum_{n=1}^{N} \alpha_{n} \varepsilon^{n}}{1+\sum_{n=1}^{N} \beta_{n} \varepsilon^{n}},
$$

where the coefficients are defined by asymptotic expansions $(2.11)$ and $(2.22)$ of the function $\bar{q}(\varepsilon)$ for large and small deflections respectively. As a result, we have $\alpha_{0}=0, \alpha_{1}=3 J_{0} / 16 \approx 0.42, \alpha_{2}=\alpha_{1} \beta_{1}$, $\beta_{1}=\alpha_{1}, \beta_{2}=\alpha_{2}, \beta_{3}=\alpha_{3}=0, \beta_{4}=\alpha_{4}=1 / 0.77 \approx 1.3$. The approximate formula has the final form

$$
\bar{q}_{0}(\varepsilon)=\frac{0.42 \varepsilon+0.176 \varepsilon^{2}+1.3 \varepsilon^{4}}{1+0.42 \varepsilon+0.176 \varepsilon^{2}+1.3 \varepsilon^{4}}
$$

The corresponding curve 1 is presented in Fig. 3. It is in a very good agreement with numerical (curve 2 ) and experimental data in the entire range of deflection amplitude. 


\section{Combined load}

Lateral perturbation point load is used to analyse the sensitivity of axially compression-loaded cylindrical shells to imperfections and estimate the lower bound of buckling value of the main load, see [23, 24, 25]. Here we consider a combination of external pressure with lateral concentrated force applied to spherical shell. We rely on asymptotic formulae obtained in [10] for the case of spherical shell under concentrated load $Q$ without external pressure, which may be presented as

$$
\frac{w_{0}}{h}=\frac{32 \bar{Q}^{2}}{J_{0}^{2} \beta}+\frac{3 \pi \bar{Q}}{4 \beta}
$$

where

$$
\bar{Q}=Q \frac{R \beta^{2}}{3 \pi E h^{3}}
$$

The first term of this formula is asymptotic solution for large deflections [10] and is actually very close to solutions obtained in [14] and [26]. The second one corresponds to the linear solution for small deflections.

The formula is valid for the entire range of deflection amplitude. Introducing parameter $\varepsilon$ according to (2.2) and solving quadratic equation for load parameter we have

$$
\bar{Q}_{0}=-0.5 a_{1}+\sqrt{0.25 a_{1}^{2}+b_{1}}
$$

where $\bar{Q}_{0}$ denotes the load parameter $\bar{Q}$ when the external pressure is equal to 0 , and

$$
a_{1}=\frac{3 \pi J_{0}^{2}}{128}, \quad b_{1}=\frac{J_{0}^{2}}{16 \varepsilon^{2}}
$$

In case of combined load and large deflections we have the following asymptotic result

$$
\bar{Q}=\frac{4}{3 \varepsilon^{2}}(0.42 \varepsilon-\bar{q})
$$

In addition, we have the obvious condition $\bar{Q} \rightarrow 0$ as $\bar{q} \rightarrow 1$. Using this observation along with asymptotic expansion (3.5) for large deflections, and equation (3.3) if $\bar{q} \rightarrow 0$ we can construct the solution for load combination, which is an approximation for arbitrary deflection amplitude and parameter $\bar{q}$

$$
\bar{Q}=\frac{4}{3 \varepsilon^{2}}\left(\bar{q}_{0}-\bar{q}\right)\left[1+\left(1-\bar{q}^{2}\right)\left(\frac{0.75 \varepsilon^{2} \bar{Q}_{0}}{\bar{q}_{0}}-1\right)\right]
$$

where $\bar{Q}_{0}$ and $\bar{q}_{0}$ are defined by $(2.24)$ and (3.3) respectively.

The approximation (3.6) accumulates all of the above mentioned properties. First of all, formula (3.6) yields the asymptotic behaviour (3.5) obtained for the combined load. This approximation is valid for large deflection. For small deflections we satisfied the condition $\bar{Q} \rightarrow 0$ as $\bar{q} \rightarrow 1$. If $\bar{Q}=0$, then we have $\bar{q}=\bar{q}_{0}$ from (3.6), which corresponds to formula (2.24) obtained for the entire range of deflection amplitude for the shell in absence of concentrated load. Similar, in case of $\bar{q}=0$, we have obtained $\bar{Q}=\bar{Q}_{0}$ given by the formula (3.3) which is also suitable for entire range of deflections if external pressure is absent, for more details see [10]. The load-deflection diagram can be obtained from (3.6) for the given external pressure $\bar{q}$.

The limiting point on the diagram yields a buckling load $\bar{Q}_{*}$.

\section{Numerical results and discussion}

The approximation of the solution in the form (3.6) is heuristic and therefore needs validation by another method. For this purpose we used ANSYS mechanical software (version ANSYS 13.0, customer 298728). 


\subsection{ANSYS model}

For the finite element (FE) shell model generation the standard ANSYS FE library element SHELL 281 is used. This element is intended to be used for shells with small and moderate thickness. The element has one additional node in the middle of every edge, which allows creating models with lower number of elements. The FE shell model is regular in both directions, every element is square in plane, except for several elements in the middle part of the shell. The average element edge size is $35 \mathrm{~mm}$. The number of elements is about 4800. Numerical analysis was carried out in geometrically nonlinear formulation. Solution is realized within the standard geometrically non-linear options implemented in ANSYS.

We examined a hemisphere with boundary conditions corresponding to hinge support: for the radial and tangent displacements zero boundary conditions are imposed on the entire circumference. Shell model has the following geometrical characteristics: the radius of mid-surface is $R=1000 \mathrm{~mm}$; the thickness is $h=5 \mathrm{~mm}$, so that $(R / h=200)$. Material properties are specified as follows: the Young modulus $E=2$ $\mathrm{GPa}$, and Poisson's ratio $\nu=0.3$.

ANSYS FE model is shown in the Fig. 5. The deformed spherical shell has just reached the stage before buckling starts. Here the corresponding pressure parameter is $\bar{q}=0.2$, the concentrated load parameter is $\bar{Q}=0.341$, and the deflection amplitude is $w_{0} / h=1.56$.

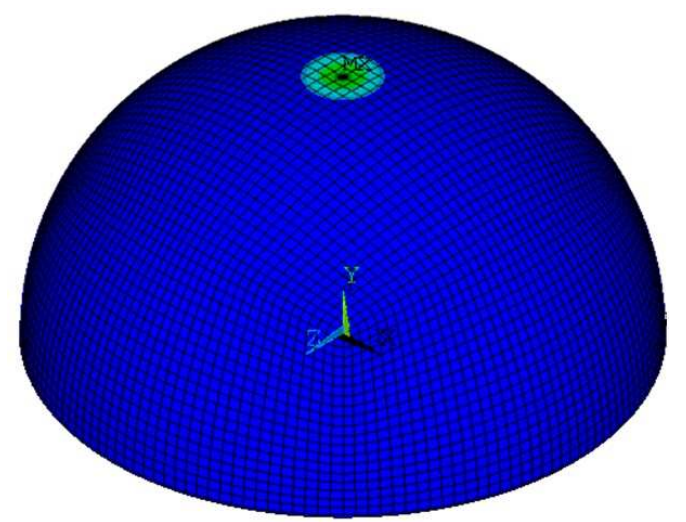

Figure 5: FE model and deformed shell under external pressure and concentrated load just before buckling.

\subsection{Discussion of the results}

The load-deflection diagram sketched according to (3.6) is shown in Fig. 6 (curve 1) for external pressure parameter $\bar{q}=0.2$. There are two special points in the graph. The first one corresponds to maximum concentrated load parameter $\bar{Q}_{*}$. It matches the shell buckling in case when the load is given and remains constant while shell is buckling (for example, dead weight). The second point with deflection amplitude $\bar{w}_{*}$ corresponds to the load parameter $\bar{Q}=0$. This case is related to the local lateral kinematic perturbation when the deflection is given (for example, loading by screw with one side constraint of the shell surface). On the other hand, this equilibrium state corresponds to post-buckling behavior of the shell under external pressure described by formula (2.24) and represented by curve 1 in the Fig. $3(\bar{Q}=0)$. Analyzing this type of perturbation in Fig. 3 one can conclude that the shell is significantly less sensitive to kinematic perturbations compared to initial deflections. For example, buckling pressure corresponding to kinematic perturbation with deflection amplitude $w_{0} / h=1$ is about $70 \%$ of classical value while initial geometrical imperfections with the same amplitude can decrease buckling pressure up to $30 \%$ of classical value [27] because deflections are increasing significantly with pressure before buckling while loading the imperfect shell by external pressure.

Numerical solution obtained using ANSYS is represented by curve 2 in Fig. 6 . There is good agreement between numerical and analytical results for both main buckling parameters including the maximum concentrated load $\bar{Q}_{*}$ and the kinematic perturbation $\bar{w}_{*}$.

In the next Fig. 7 the buckling combinations of concentrated load and external pressure are shown (curve 1 - analytical solution based on (3.6), curve 2 - numerical result). Analytical solution obtained by 


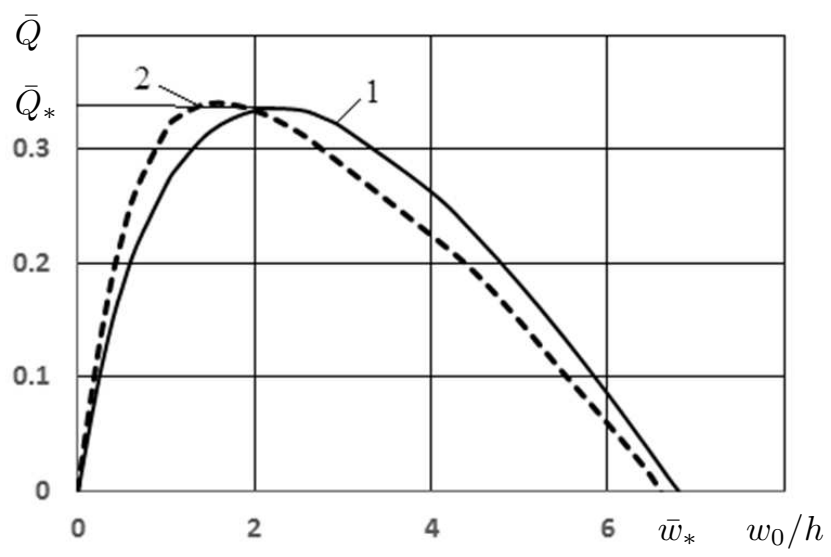

Figure 6: Load deflection diagram obtained for external pressure parameter $\bar{q}=0.2$.

Sabir [6] is represented by curve 3. The suggested formula (3.6) yields much better approximation of the numerical results compared to Sabir's solution.

If the corresponding deflection amplitude at buckling load $\bar{Q}_{*}$ is large enough, we can use the simple asymptotic formula (3.5) and derive the approximation for the maximum of concentrated load parameter

$$
\bar{Q}_{*}=\frac{0.58}{\bar{q}} .
$$

The associated curve 4 is represented in Fig. 7.

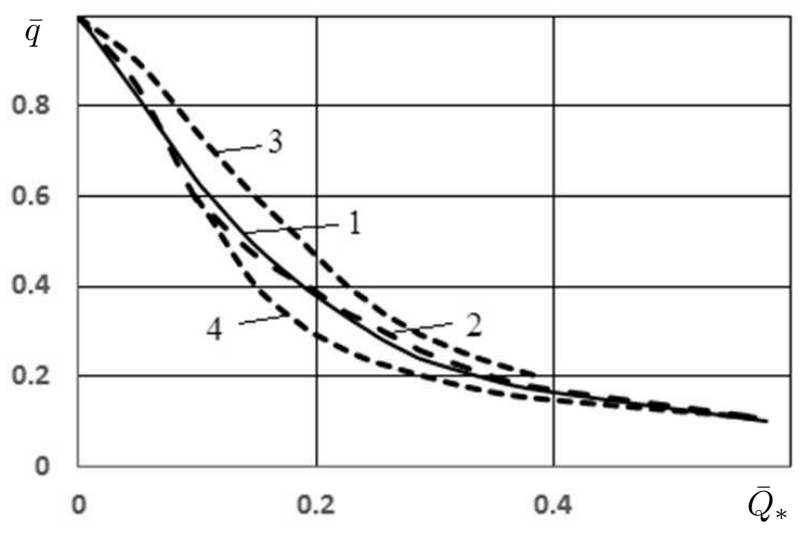

Figure 7: Buckling load combination diagram.

Babenko and Prichko [15] studied the problem using both experimental and numerical methods. Numerical results are very close to those obtained above using ANSYS. Their lower bound of experimental results coincides with our asymptotic solution (3.6), while their upper bound is close to the solution obtained by Sabir. The methodology of manufacturing shell specimens from copper was the same as in [9].

Some results of calculation are presented in Table 1. It is observed that solution of Sabir [6] is an upper bound estimate. Maximum relative error is reached by large deflections and it is about $20 \%$. This difference is due to linear formulation employed in [6]. If one used linear equations in our asymptotic analysis of the inner boundary layer (Fig. 2) for large deflections, the coefficient $J_{0}$ would be $J_{0}=2 \sqrt{2}$ (instead of $J_{0}=2.23$ ), which is exceeds the one obtained through a more accurate nonlinear analysis by $20 \%$. A simple formula (4.1) is valid for small external pressure parameter $\bar{q} \leq 0.15$. It is also clear from the data of Table 1, that the asymptotic formula (3.6) yields result with very good accuracy if $\bar{q} \leq 0.5$ covering all practical cases. 


\begin{tabular}{|c|c|c|c|c|c|}
\hline $\bar{q}$ & 0.1 & 0.2 & 0.3 & 0.4 & 0.6 \\
\hline Sabir & - & 0.386 & 0.283 & 0.227 & 0.149 \\
\hline ANSYS & 0.575 & 0.341 & 0.257 & 0.194 & 0.098 \\
\hline Formula (3.6) & 0.580 & 0.335 & 0.245 & 0.189 & 0.111 \\
\hline Formula (4.1) & 0.58 & 0.290 & 0.193 & 0.145 & 0.097 \\
\hline
\end{tabular}

To estimate the sensitivity of spherical shell to the additional concentrated load we consider the ratio of the load to resultant pressure on the spherical cap with base radius $r_{0}$

$$
\frac{Q}{\pi r_{0}^{2} q}=\frac{3 \bar{Q}}{k^{2} \bar{q}},
$$

where

$$
k^{2}=\frac{2 \beta r_{0}^{2}}{R h},
$$

is slenderness of a shell. For a complete sphere or a hemisphere we have

$$
k^{2}=\frac{2 \beta R}{h} .
$$

If the ratio is not small, we have to consider the structure under combined load and can obtain buckling loads using (3.6). If the concentrated load is small and not completely defined, it can be considered as additional perturbation. Let us assume for example that the concentrated load is only $2 \%$ of the resultant pressure. Then

$$
\frac{Q}{\pi r_{0}^{2}}=\frac{3 \bar{Q}}{k^{2} \bar{q}}=0.02 .
$$

The curve 1 in Fig. 8 shows the corresponding buckling pressure parameter versus shell slenderness. The curve exhibits significant sensitivity of the structure to concentrated lateral load perturbation. The sensitivity is increasing dramatically with increasing of shell slenderness. It is similar to the sensitivity to initial geometrical imperfection. This is reasonable because initial deflections lead not only to change of the structure shape, but also yield the effect of additional inward load, which seems more important as a factor that decreases buckling pressure. Curve 2 in the Fig. 8 corresponds to the concentrated load which is only $1 \%$ of pressure resultant. Both curves in Fig. 8 are similar to limiting curve in Fig. 1.

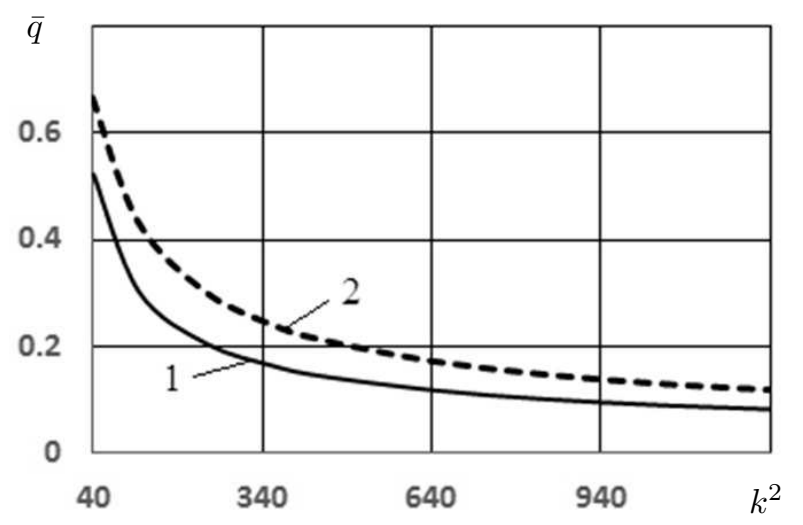

Figure 8: Buckling external pressure parameter versus shell slenderness.

\section{Conclusions}

The approximate analytical solution is suggested for spherical shell buckling load combination of uniform external pressure and lateral concentrated load. The asymptotic formula (2.24) is suggested for postbuckling behaviour of a thin spherical shell under uniform external pressure. It is in good agreement 
with numerical results. The asymptotic approach is expanded to the case of combined load. The result is validated by ANSYS. The influence of kinematic and force perturbations on the shell buckling under external pressure is analyzed. The sensitivity of the structure to concentrated load perturbation is revealed to be similar to sensitivity of the structure to initial geometric imperfections. Among further directions of this research we mention analysis of sensitivity of the shell to different types of external and internal perturbations.

\section{Acknowledgement}

A.E. would like to thank Keele University for hospitality during his visit, supported by the Faculty of Natural Science Research Support Fund. Fruitful discussions with Dr E. Nolde are also gratefully acknowledged.

\section{References}

1. Zoelly R. Über ein Knickungsproblem an der Kugelschale, Thesis, Zürich, 1915.

2. Timoshenko SP, Gere JM. Theory of Elastic Stability, 2nd Ed., McGraw Hill, New York, 1961; $512-519$.

3. Wunderlich, W., Albertin U. Buckling Behaviour of Imperfect Spherical Shells. Int. J. Non-Lin. Mech. 2002; 37: 589-604.

4. Samuelson LA, Eggwertz S. (Eds.) Shell Stability Handbook, Elsevier, London, 1992.

5. Koiter WT. The Nonlinear Buckling Problem of a Complete Spherical Shell Under Uniform External Pressure. Proc. Kon. Nederl. Acad. Wet. Amsterdam. 1969; B72: 40-123.

6. Sabir AB. Large deflections and buckling behavoiur of a spherical shell with inward point load and uniform external pressure. J. Mech. Eng. Sci. 1964; 6(4): 394-404.

7. Ashwell DG. On Large Deflection of a Spherical Shell with an Inward Point Load. In: Proc. IUTAM Symp. on Theory of Thin Elastic Shells, Delft, August 1959. North Holland, Amsterdam, 1959; 43-63.

8. Ranjang FA, Steele CR. Large Deflection of Deep Spherical Shells Under Concentrated Load. In: Proc. AIAA J/ASME 18th Structures. Structural Dynamics and Materials Conference. San Diego, California, USA, 1977. Technical paper no 77-411, 269-278.

9. Babenko VI, Prichko VM. Stability of Shallow Spherical Shells under Combined Load. Doklady Akad Nauk USSR Ser. A. 1985; 6: 28-30.

10. Evkin AY. Large Deflections of Deep Orthotropic Spherical Shells Under Radial Concentrated Load: Asymptotic Solution. Int. J. Solids Struct. 2005; 42: 1173-1186.

11. Evkin AY. A New Approach to the Asymptotic Integration of the Equations of Shallow Convex Shell Theory in the Postcritical Stage, PMM (J. Appl. Math. Mech.) 1989; 53(1): 92-96.

12. Evkin A., Kalamkarov A. Analysis of Large Deflection Equilibrium States of Composite Shells of Revolution. Part 1. General Model and Singular Perturbation Analysis, Int. J. Solids Struct. 2001a; 38: 8961-8974.

13. Evkin A., Kalamkarov A. Analysis of Large Deflection Equilibrium States of Composite Shells of Revolution. Part 2. Applications and Numerical Results, Int. J. Solids Struct. 2001b; 38: 8975-8987.

14. Pogorelov AV. Bendings of Surfaces and Stability of Shells, Providence, R.I.: American Math. Soc., 1988.

15. Babenko V., Prichko F. Load Carrying Diagram of Spherical Caps under External Pressure. Doklady Akad Nauk SSSR, 1985; 10: 21-24. 
16. Gabril'yants AG, Feodos'yev VI. On axisymmetric equilibrium modes of an elastic spherical shell subjected to a uniformly distributed pressure, PMM, 1961; 25(6): 1091-1101

17. Thompson, JMT. The Elastic Instability of a Complete Spherical Shell. Aero. Quart., 1962; 13: 189-202.

18. Thompson, JMT. The Rotationally-Symmetric Branching Behavoiur of a Complete Spherical Shell. Koninklijke Nederland Akademie van Welenschapen Proc. Ser. B, 1964; 67: 295-311.

19. Evkin, AY, Korovaitsev, AV. Asymptotic Analysis of the Transcritical Axisymmetric State of Stress and Strain in Shells of Revolution under Strong Bending. Mech. Solids, 1992; 27(1): 121-129.

20. Andrianov, IV, Awrejcewicz, J., Manevitch, LI. Asymptotical Mechanics of Thin-Walled StructuresL A Nandbook, Springer-Verlag, Berlin, 2004.

21. Andrianov, IV, Awrejcewicz, J., Danishevs'kyy, VV, Ivankov, AO. Asymptotic Methods in the Theory of Plates with Mixed Boundary Conditions, Wiley, New York, 2014.

22. Baker G., Graves-Morris P. Padé approximants, Addison-Wesley Publ. Co, London, 1995.

23. Evkin AY, Krasovsky, VL, Manevich LI. Stability of Longitudinally Compressed Cylindrical Shells under Quasi-Static Local Disturbances, Mech. Solids, 1978; 13(6): 83-88.

24. Thompson, JMT. Advances in Shell Buckling: Theory and Experiments. Int. J. Bifurc. and Chaos, 2005; 25: 1530001.

25. Kriegesmann, B, Hilburger, M, Rolfes, R. The Effects of Geometric and Loading Imperfections on the Response and Lower-Bound Buckling Load of a Compression-Loaded Cylindrical Shell. AIAA Paper 2012-1864, Proc. 53 ${ }^{\text {rd }}$ AIAA/ASME/ASCE/AHS/ASC Structures, Structural Dynamics and Materials Conference, April 2012, Honolulu, HI.

26. Holst JMFG, Calladine CR. Inversion Problems in Elastic Thin Shells, Europ. J. Mech. A Solids, 1994; 13(4): 3-18.

27. Starlinger A., Rammerstorfer FG., Auli W. Beulen und Nachbeulverhalten von dünnen verrippten und unverrippten Kugelschalen unter Außendruck. ZAMM, 1988; 68(4): 257-260. 